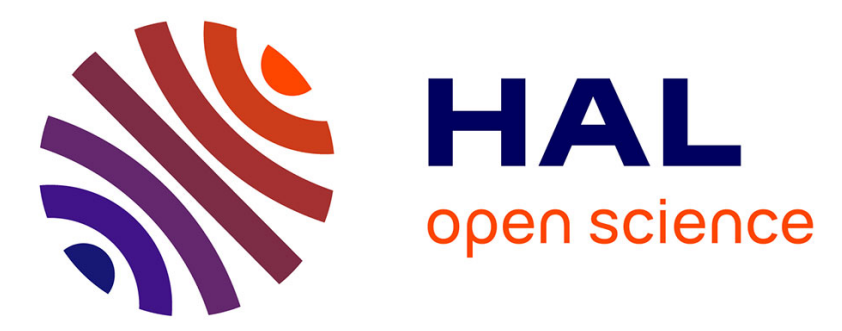

\title{
N-atom Production at High Electric Fields: E-FISH and TALIF Experiments for Understanding Fast Ionization Wave Kinetics
}

Tat Loon Chng, Inna S Orel, Igor V Adamovich, Nikolay A Popov, Svetlana Starikovskaia

\section{To cite this version:}

Tat Loon Chng, Inna S Orel, Igor V Adamovich, Nikolay A Popov, Svetlana Starikovskaia. Natom Production at High Electric Fields: E-FISH and TALIF Experiments for Understanding Fast Ionization Wave Kinetics. Proc. of AIAA Scitech 2020 Forum Session: Diagnostics, Jan 2020, Orlando, United States. hal-03042116

\section{HAL Id: hal-03042116 https://hal.science/hal-03042116}

Submitted on 5 Dec 2020

HAL is a multi-disciplinary open access archive for the deposit and dissemination of scientific research documents, whether they are published or not. The documents may come from teaching and research institutions in France or abroad, or from public or private research centers.
L'archive ouverte pluridisciplinaire HAL, est destinée au dépôt et à la diffusion de documents scientifiques de niveau recherche, publiés ou non, émanant des établissements d'enseignement et de recherche français ou étrangers, des laboratoires publics ou privés. 


\title{
N-atom Production at High Electric Fields: E-FISH and TALIF Experiments for Understanding Fast Ionization Wave Kinetics
}

\author{
Tat Loon Chng, ${ }^{1}$ Inna S. Orel, ${ }^{2}$ \\ CNRS, Ecole Polytechnique, University Paris-Sud, Observatoire de Paris, Institut Polytechnique de Paris, \\ Palaiseau, 91128, France \\ Igor V. Adamovich ${ }^{3}$ \\ Nonequilibrium Thermodynamics Laboratories, The Ohio State University, Columbus, OH 43210, USA \\ Nikolay A. Popov ${ }^{4}$ \\ Moscow State University, Moscow, 119991, Leninsky gory, Russia
}

Svetlana M. Starikovskaia ${ }^{5}$

CNRS, Ecole Polytechnique, University Paris-Sud, Observatoire de Paris, Institut Polytechnique de Paris, Palaiseau, 91128, France

\begin{abstract}
This work forms part of a larger effort to develop a suite of diagnostics for making measurements in non-equilibrium, nanosecond pulse discharges, so as to facilitate an improved understanding of the plasma kinetics. Electric field induced second harmonic (E-FISH) generation, is used to probe the electric field in a fast ionization wave, nanosecond pulse discharge in pure $\mathrm{N}_{2}$ at a pressure of 20 mbar. The field evolution during the fast ionization wave development is clearly captured in the form of three distinct phases. An initial field overshoot ahead of the front to about $10.5 \mathrm{kV} / \mathrm{cm}$ (or about 2 kTd), followed by a field drop as the wave traverses the measurement location, and finally a subsequent rise as a quasi-steady state regime is established. TALIF measurements of $\mathrm{N}$-atom density are also performed with a view to understanding the impact of the reduced electric field on the consequent atomic species production. These measurements are limited to the post-discharge phase, mainly due to the poor signal to raise ratio associated with the lower atomic densities and strong fluorescence-overlapping plasma emission. A relatively low peak $\mathrm{N}$-atom density of about $5.5 \times 10^{12} \mathrm{~cm}^{-3}$ is obtained, in line with the low specific deposited energy of this discharge $(0.01 \mathrm{eV} / \mathrm{molecule})$. Finally, attempts to model this plasma show that the results of simulations are strongly influenced by the radial non-uniformity of the discharge.
\end{abstract}

\section{Introduction}

$\mathrm{T}$ The primary objective of this study is to examine the electric field evolution in a fast ionization wave produced by a nanosecond pulse discharge. Measurements are performed using electric field induced second harmonic (E-FISH) generation, a recently redeveloped laser-based diagnostic with excellent temporal (sub-ns) and spatial (sub-mm) resolution [1]. The discharge apparatus used to study the wave front development is a relatively high aspect-ratio quartz tube oriented (along its length) between two electrodes, a configuration which has come to be commonly known in the literature as a fast ionization wave (FIW) discharge. Typical operating pressures of these discharges range from $1-100$ mbar, with reported wave speeds as high as two-thirds the velocity of light.

An important aspect of FIWs is the presence of high-energy electrons, which serve as an initial source of preionization, and consequently lead to a spatially uniform development of the discharge [2]. This spatial homogeneity is one of the defining features of FIW discharges, and often facilitates the comparison of both numerical and experimental data. The use of over-voltage nanosecond pulses with a fast voltage rise rate $(\sim 1$

\footnotetext{
${ }^{1}$ Postdoctoral researcher, Laboratory of Plasma Physics, AIAA member.

${ }^{2}$ Graduate student, Laboratory of Plasma Physics.

${ }^{3}$ Professor, Dept. of Mechanical and Aerospace Engineering, Associate Fellow AIAA.

${ }^{4}$ Leading scientific researcher, Skobeltsyn Institute of Nuclear Physics.

${ }^{5}$ Senior researcher, Laboratory of Plasma Physics, AIAA member.
} 
$\mathrm{kV} / \mathrm{ns}$ ) produces a uniform wave front with an extremely high reduced electric field $(E / N)$ on the order of a few $100 \mathrm{Td}$, and has since evolved into an intriguing subject of research from both a physical and application standpoint.

Given the above, it is therefore important to understand the electric field evolution in a FIW. Over the past two decades, the main mode of such measurements have been in the form of electrical probes such as back current shunts and capacitive dividers [3-5]. The latter in particular, has very good time resolution ( $1 \mathrm{~ns})$, is virtually non-intrusive, and provides a qualitative description of the electric field behavior during the passage of the FIW. The spatial resolution of the capacitive probe however, is relatively modest $(\sim 1 \mathrm{~cm})$ and this limits the accuracy of peak field estimates, especially when the wave speed is high. In addition, capacitive probes are only capable of measuring the longitudinal component of the electric field vector (i.e. parallel to length of the discharge tube). More recently, electro-optic probes (based on the Pockels effect) capable of resolving dual components of the electric field vector have been successfully employed in an atmospheric pressure plasma jet [6,7]. The resulting measurements are well localized in both time $(\sim 1 \mathrm{~ns})$ and space $(\sim 1 \mathrm{~mm})$, and may yet prove to be a promising alternative to electrical probes. The main limitation of such as a sensor is that it is physically intrusive and any perturbation effects on the plasma need to be carefully assessed (and possibly accounted for) before a reliable electric field measurement is obtained.

The foregoing discussion forms the motivation for attempting to make electric field measurements using the E-FISH diagnostic. Apart from its exceptional temporal and spatial resolution, this laser-based method is simple to use (only one laser beam is required) and versatile in its implementation (does not require any spectroscopic resonance). This study is also part of a broader endeavor to understand the effect of the reduced electric field on the consequent atomic species production in nanosecond pulse discharges. To this end, two-photon laser induced fluorescence (TALIF) is used to monitor the time evolution of the $\mathrm{N}$-atom production in the early to late discharge afterglow.

\section{Experimental Setup}

\section{A. Discharge Specifics}

A schematic of the discharge cell geometry used in this work is presented in Fig. 1a). This configuration is similar in principle to that used in Ref. [8]. A cylindrical quartz tube with a nominal length of $92 \mathrm{~mm}$ and an internal and external diameter of $20 \mathrm{~mm}$ and $24 \mathrm{~mm}$ respectively sits between two hollow, semi-conical electrodes with an interelectrode gap distance of about $80 \mathrm{~mm}$. A cylindrical copper screen surrounds the quartz tube in an annular fashion, and serves as a grounded surface which facilitates both the initiation and propagation of the FIW. Pure $\mathrm{N}_{2}$ gas enters and exits the tube via two glass side arms at a flow rate of $10 \mathrm{sccm}$ by means of a Brooks Instruments 5850TR mass flow controller. The static pressure in the capillary is kept constant at 20 mbar based on previous work, which indicated this as a good pressure for reducing parasitic emission in the side tubes, without severely compromising the specific deposited energy to the plasma. Pressure measurements are made using a pair of CMR 362 capacitive pressure sensors (from Pfeiffer Vacuum SAS), each positioned at an approximately equidistant location upstream and downstream of the discharge tube. The operating pressure was determined by taking the average of both these sensor readings. This approach for pressure quantification is chosen due to difficulties associated with mounting a pressure transducer directly onto the quartz tube. 
a)
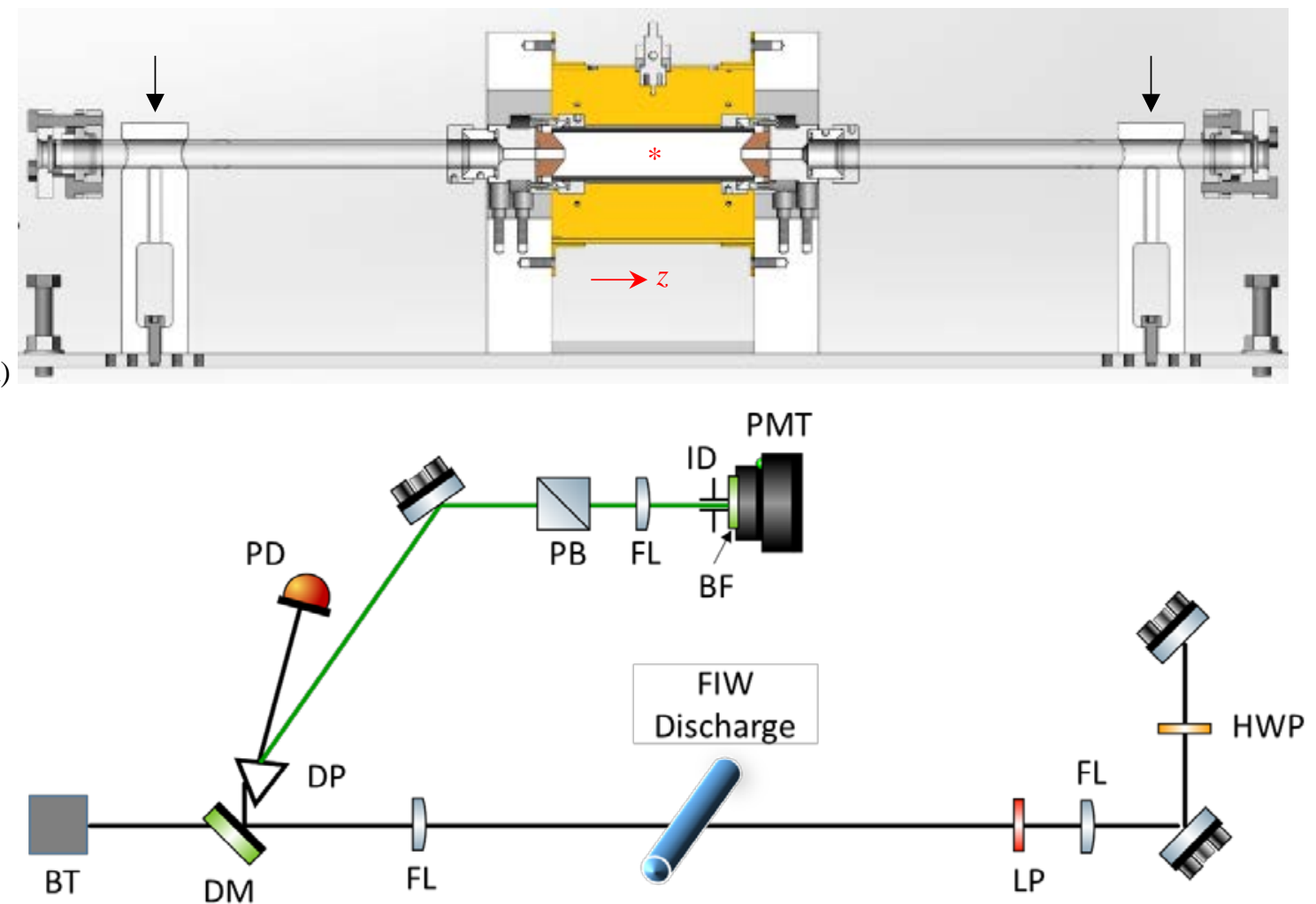

b)

c)
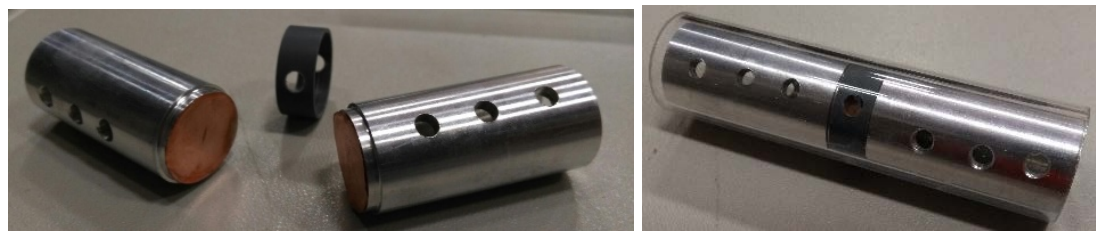

Fig. 1 a) Schematic of the discharge cell setup used in this study. (The measurement location for both the E-FISH and TALIF experiments is indicated by a red asterisk and the location of the gas flow inlets/outlets (hidden from view) are indicated with black arrows.) b) Optical layout of the E-FISH experiment. c) Pictures of the inserts and the modified quartz tube geometry used for the calibration experiments.

High voltage nanosecond pulses are delivered by a FPG 12-1NM high-voltage generator (HVG) manufactured by FID GmbH. Positive polarity, $9.3 \mathrm{kV}$ amplitude voltage waveforms with a rise time of $4 \mathrm{~ns}$ and a full width at half maximum (FWHM) of 30 ns are produced by this high voltage source at a repetition rate of $10 \mathrm{~Hz}$. The output of this high-voltage generator is connected to one of the electrodes (henceforth termed high-voltage electrode) via a $50 \mathrm{ohm}$ impedance RG213 coaxial cable. A second such coaxial cable of identical length is connected to the remaining electrode (low-voltage electrode) but is kept ungrounded at a floating potential. The shielding of both these coaxial cables is connected to the copper screen. Due to an impedance mismatch between the high voltage pulses and the discharge (viz. electrical load), each pulse delivered by the HVG is reflected twice, once from the high-voltage electrode and a second time from the HVG itself. This leads to a sequence of 3 incident pulses seen by the discharge (see figure 2), each separated from the next by about 250 ns. Custom-made, calibrated back current shunts (BCS) are installed at mid-length of both coaxial cables for measuring the amplitude of the high voltage pulses and the transmitted current, thereby facilitating calculations of the specific energy coupled to the plasma [4]. 

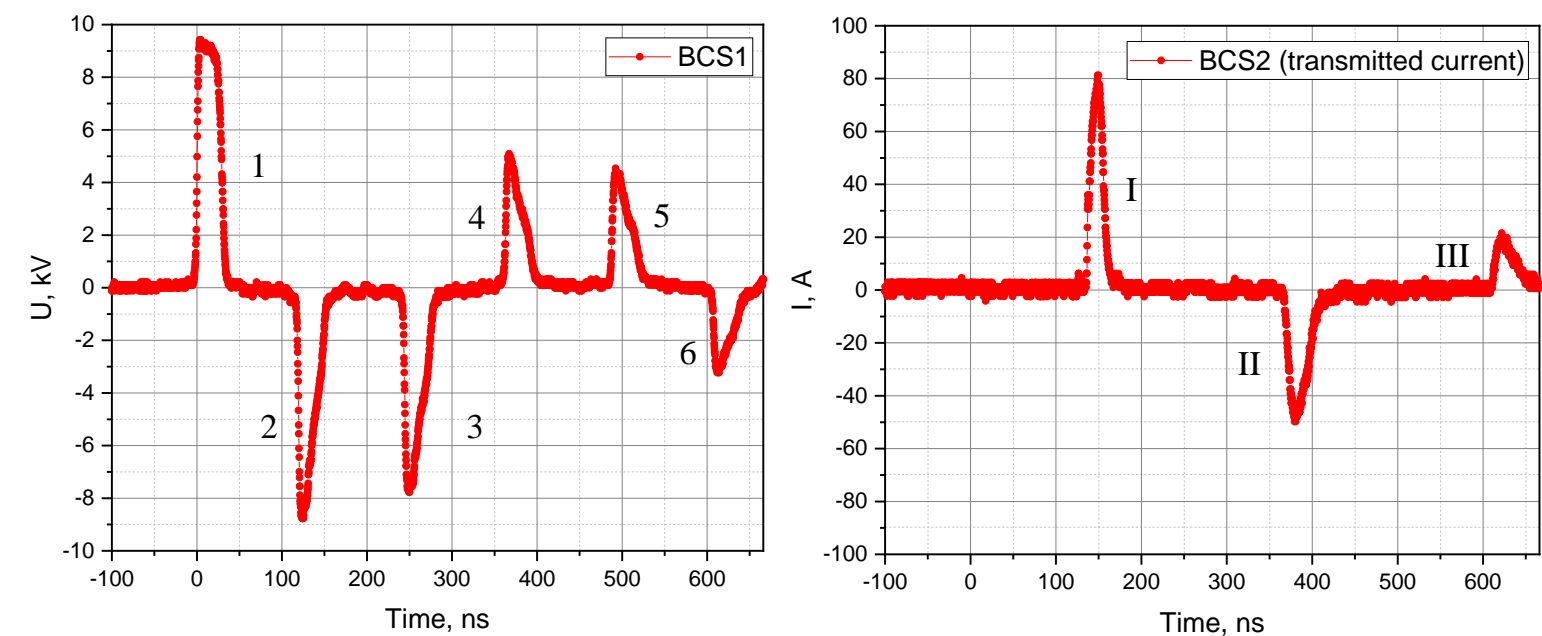

Fig. 2 Typical signals acquired from the calibrated BCSs for pure $\mathrm{N}_{2}$ at a pressure of 20 mbar. BCS1 captures the incident and reflected pulses delivered to the discharge tube, while BCS2 measures the transmitted pulses. Pulses labelled 1, 3 and 5 in the BCS1 waveform are the incident pulses seen by the discharge tube, while pulses 2, 4 and 6 are the corresponding reflected pulses. Transmitted pulses I, II and III of the electrical current are measured after the discharge cell by BCS2.

\section{B. E-FISH Specifics}

An EKSPLA PL2241B picosecond mode-locked Nd:YAG laser which delivers $1064 \mathrm{~nm}, 30$ ps pulses, at a repetition rate of $10 \mathrm{~Hz}$, is used for the E-FISH experiments. The maximum energy per pulse of this laser system is $80 \mathrm{~mJ}$ but only $6 \mathrm{~mJ}$ of horizontally polarized light is used for the experiments. The optical layout of the EFISH experiments is shown in Fig. 1b) and is very similar to that used in [9]. As shown in Fig. 1a), the $1064 \mathrm{~nm}$ beam is focused into the center of the quartz tube, at its mid-length and approximately along its horizontal plane of symmetry, using an $f=20 \mathrm{~cm}$ plano-convex spherical lens. The beam propagation direction is parallel to the optical table and orthogonal to the length of the tube. A second identical lens collimates both the coherent 532 $\mathrm{nm}$ second harmonic signal emanating from the measurement volume and the residual $1064 \mathrm{~nm}$ light. The confocal beam parameter of $1064 \mathrm{~nm}$ is calculated to be approximately $1 \mathrm{~mm}$ based on a Gaussian beam approximation. The $1064 \mathrm{~nm}$ probe beam and the $532 \mathrm{~nm}$ signal is then separated, first by a dedicated $532 \mathrm{~nm}$ reflecting, 1064 $\mathrm{nm}$ transmitting dichroic mirror, followed by a dispersive equilateral prism. The horizontally polarized component of the $532 \mathrm{~nm}$ signal is isolated using a broadband polarizer and focused via a $10 \mathrm{~cm}$ focal length lens onto a photomultiplier (PMT). The residual $1064 \mathrm{~nm}$ beam is directed onto a Thorlabs DET10A photodiode with a response time of $1 \mathrm{~ns}$. The photodiode signal serves as both a monitor of the laser intensity (assuming negligible conversion or depletion of the $1064 \mathrm{~nm}$ probe beam) as well as an indication of the actual time instant of the EFISH measurement (since the photodiode and PMT signals are separated by a fixed time delay). A $600 \mathrm{MHz}$ bandwidth LeCroy WaveRunner 64Xi-A oscilloscope with a resolution of 200 ps is used to record the BCS, photodiode and PMT waveforms.

Due to a significant $30 \mathrm{~ns}$ timing jitter between the arrival of the laser pulse and the initiation of the discharge, on-scope averaging is not possible. Instead, about 40000 sets of single shot waveforms are acquired and timesequenced during the post-processing stage based on the timing of the photodiode signals and the BCS waveforms. (The latter is a reliable indication of the discharge timing.) This 'time-binning' procedure has been observed in previous work [10]. The resulting shot statistics were found to be adequate to yield a measurement time resolution of 200 ps (i.e. equaling the time resolution of the oscilloscope), with a corresponding 20-250 shots per time step. Photodiode and PMT signals are time-integrated so as to provide a measure of the laser intensity and E-FISH signal respectively. These time-integrated values are averaged at each time step, and the square root of the EFISH signal, normalized by the laser-intensity is used to infer the axial component of the externally applied electric field (see eqn. (1) below).

\section{Nanosecond TALIF Specifics}

Absolute N-atom densities are obtained using TALIF by calibrating against a known amount of krypton (Kr) gas. The dye laser source and TALIF excitation scheme used are identical to that described in [11]. For these experiments, the UV probe beam (206.7 nm for $\mathrm{N}$ and $204.1 \mathrm{~nm}$ for $\mathrm{Kr}$ ) is focused with a $50 \mathrm{~cm}$ focal length, plano-convex spherical lens into the center (mid-length) of the quartz tube. In contrast to the E-FISH experiments, 
the $3 \mathrm{~mm}$ diameter TALIF probe beam propagates parallel to the length of the tube, and gives rise to a confocal beam parameter of about $1.5 \mathrm{~cm}$ based on a Gaussian beam approximation. The resulting fluorescence is collected perpendicular to the length of the tube, by means of imaging system comprising a 1 in diameter, $f=15 \mathrm{~cm}$ planoconvex spherical lens which collimates the light, followed by an identical, $f=20 \mathrm{~cm}$ lens which focuses this light into a Hamamatsu (H7422-50P) photomultiplier (PMT). The laser intensity is monitored by picking off a small fraction of the beam intensity using a quartz slide and directing this light onto a 1 ns response time, Thorlabs DET10A photodiode.

\section{Results and Discussion}

\section{A. E-FISH Experiments}

Based on a plane-wave approximation, the horizontally-polarized component of the E-FISH signal, $I_{z}^{(2 \omega)}$ may be written as:

$$
I_{z}^{(2 \omega)} \propto\left[\chi^{(3)}(2 \omega, 0, \omega, \omega) \cdot N \cdot E_{z}^{e x t} \cdot I_{z}^{(\omega)} \cdot L \cdot \operatorname{sinc}\left(\frac{\Delta k \cdot L}{2}\right)\right]^{2}
$$

In Eq. (1), $E^{\text {ext }}$ represents the electric field to be measured, $I_{z}^{(\omega)}$ is the intensity of the vertically-polarized $1064 \mathrm{~nm}$ probe laser, $\chi^{(3)}$ is the third-order nonlinear hyperpolarizability, $N$ is the gas number density, $L$ is the confocal beam parameter and $\Delta k$ is the difference between the fundamental and second harmonic wave vectors. Here, $z$ is defined as the horizontal axis, i.e. the axis parallel to the length of the discharge tube. It should be noted that the polarization of the E-FISH signal echoes the polarization of the applied electric field. The signal is also strongest when the polarization of the probe beam is maintained parallel to the polarization of the applied field [1]. Hence, capturing the $z$-component of the E-FISH signal provides information on the longitudinal electric field produced by the discharge.

Based on eqn. (1), a convenient approach to obtaining absolute electric field information in a plasma is to perform signal calibration in the presence of a known applied field. It should be emphasized, following eqn. (1), that these calibration experiments should be conducted under conditions where both the number density and the gas composition are similar to that of the plasma. For this study, the setup is modified so as to sustain an axial Laplacian field within the discharge cell. This is achieved through a set of custom-made inserts (see Fig. 1c)), which enable a pair of vertically parallel, copper plate electrodes to be placed within the quartz tube without substantially affecting the flow of gas through the cell. The distance between the two parallel plate electrodes is about $4 \mathrm{~mm}$. In this way, calibration can be performed in situ, utilizing the same optical layout for the actual experiments.

Figure 2 plots the temporal evolution of the Laplacian field produced by a $1.26 \mathrm{kV}$ high-voltage pulse together with single shot and averaged E-FISH (square-rooted) signals. The applied voltage is determined directly from the BCS waveforms and the electric field strength is then computed by dividing this voltage by the interelectrode gap distance of $4 \mathrm{~mm}$. The E-FISH signals accurately capture the shape of the high-voltage pulse, especially during the rise and plateau, and indicates that the expected quadratic dependence of the signal on the applied electric field is observed. However, the source of a slight systematic deviation on the falling section is yet unclear but could be due to the development of a very weak discharge. As such, signals corresponding to the falling edge of the HV pulse are excluded from the data used for calibration. Figure 3 shows a plot of the calibration curve obtained by directly matching the field strengths obtained from the BCSs with their respective square-rooted EFISH signals, illustrating the expected linear dependence between these two quantities. This calibration curve is used to provide absolute measurements of the field strength during the discharge as shown in fig. 4. Reasonable agreement is obtained between the field strengths measured using this approach with that obtained via the BCSs. The latter approach is based on a significant body of previous work [4,5], which has found that after the ionization wave arrives at the low-voltage electrode, the axial electric field distribution along the discharge tube is essentially uniform and is well approximated by the difference between the potentials on the electrodes divided by the discharge gap length.

The evolution of the electric field during the passage of the fast ionization wave displays 3 distinct phases: a field overshoot (above the critical breakdown threshold) ahead of the front to about $11 \mathrm{kV} / \mathrm{cm}$, followed by a drop in the field strength to near detection limit as the wave passes the measurement location, and finally a rise in the field to a second, weaker peak as the wave reaches the low voltage electrode. This pattern of development agrees very well with what has previously been observed in the literature. The initial field overshoot ahead of the front is a commonly observed phenomenon associated with fast ionization waves, and has been measured in the literature using several different techniques [15-17]. The subsequent drop in the electric field is likely to be due to both the passage of the wave as well as plasma self-shielding. The increase in the field strength observed during the $3^{\text {rd }}$ and final phase is associated with a quasi-steady state regime which occurs, along with a redistribution of 
space charge, as the ionization wave 'closes' the gap. It is at this stage that the discharge becomes a fully conductive medium.

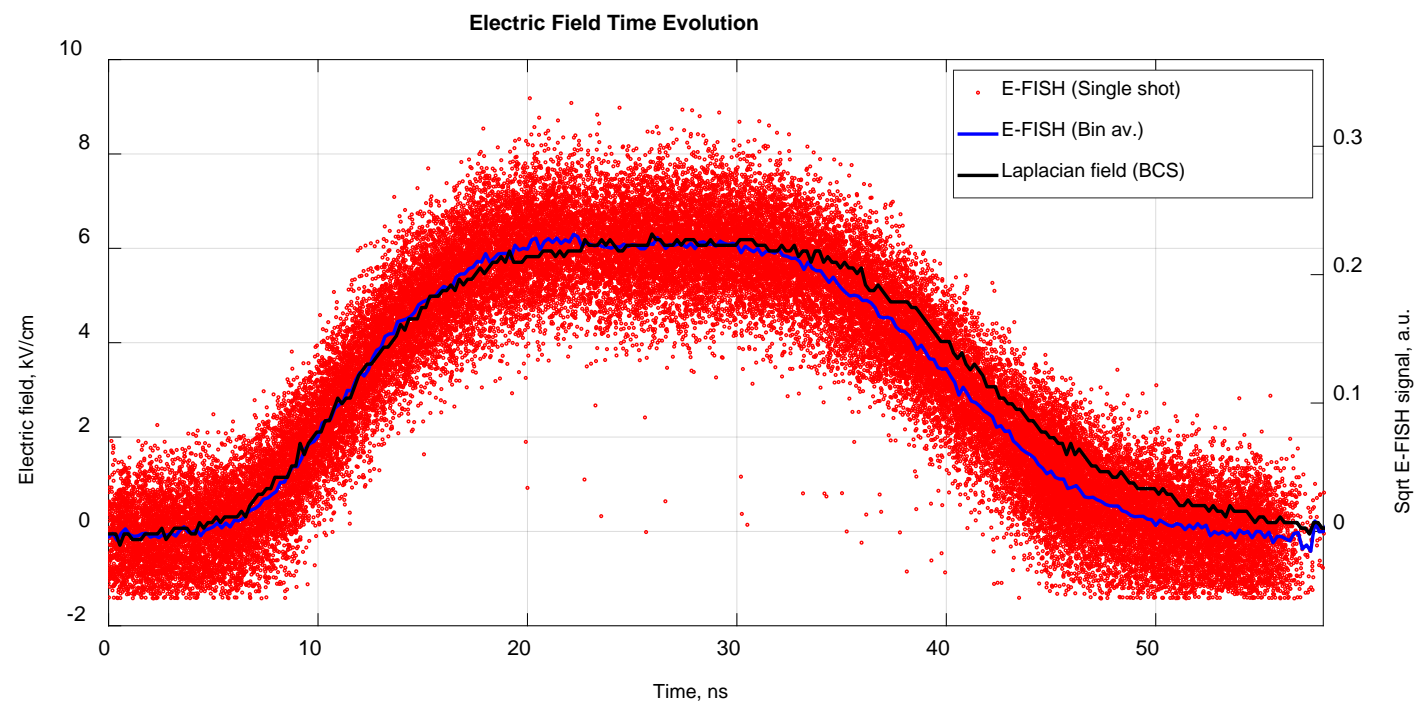

Fig. 3 Temporal evolution of the electric field plotted together with square rooted E-FISH signals (single shot and averaged) obtained from Laplacian calibration.

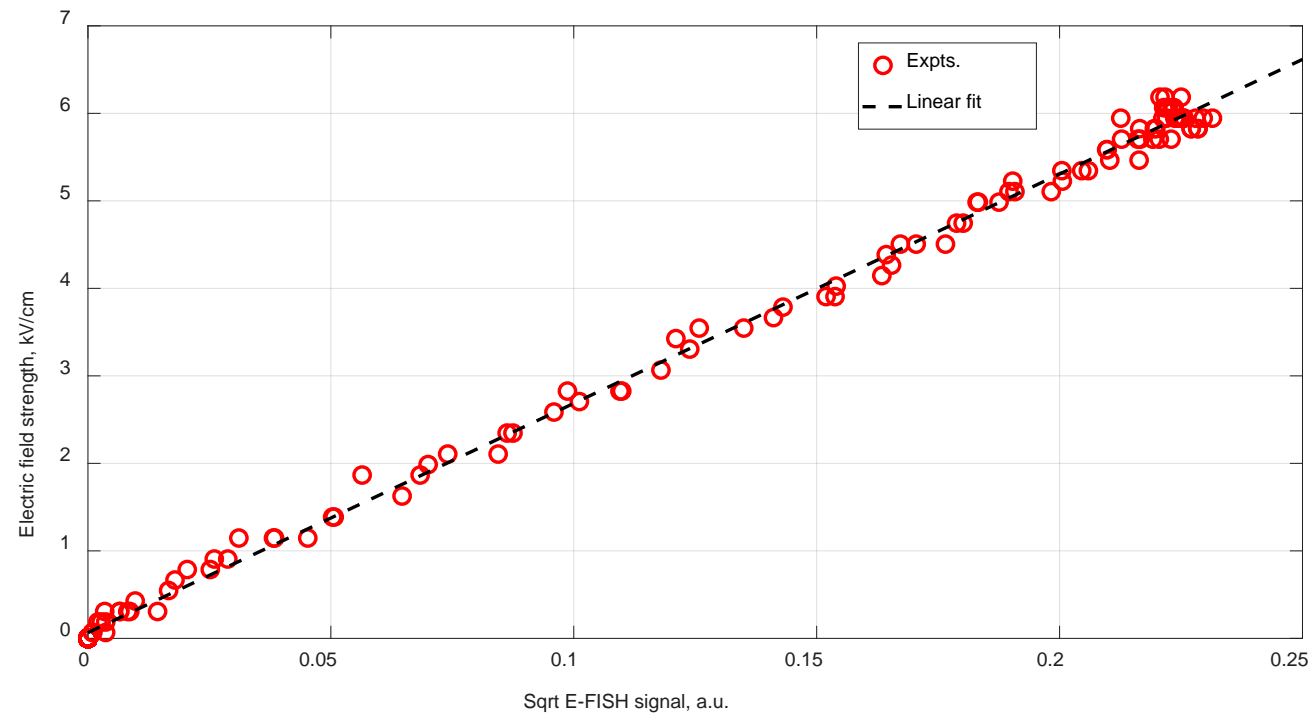

Fig. 4 Calibration curve obtained by plotting the bin averaged, square-rooted E-FISH signals against the matching field strengths obtained from the calibrated BCS waveforms shown in figure 3 .

While these results are encouraging, a few aspects remain worthy of further research. Further calibration experiments conducted at higher pressures exhibit a virtual non-dependence of the E-FISH signal on pressure. This raises the possibility that the signal generation could originate from within the quartz tube rather than the gas medium. While the E-FISH signal displays a quadratic dependence on number density (viz. pressure if the temperature is constant), any signal originating from the quartz tube would be essentially independent of the pressure. Furthermore, while the laser intensity is much weaker near the quartz tube, this effect could be compensated by the significantly higher non-linear susceptibility of quartz (see eqn. (1)).

Another drawback of the current calibration approach is that extra effort is needed to construct the custommade inserts required for sustaining the Laplacian field. This also involves disassembly of the discharge apparatus which could inadvertently lead to a source of optical alignment error. An alternative solution is to perform calibration using the second incident high-voltage pulse (for example, pulse 3 in fig. 2). After the passage of the FIW produced by the first high-voltage pulse, the interelectrode gap is essentially closed and replaced by a conductive medium. The discharge is effectively in a quasi-steady state and a good estimate of the electric field strength may be simply obtained by taking the potential difference across the two electrodes and dividing this by the corresponding interelectrode gap distance. 


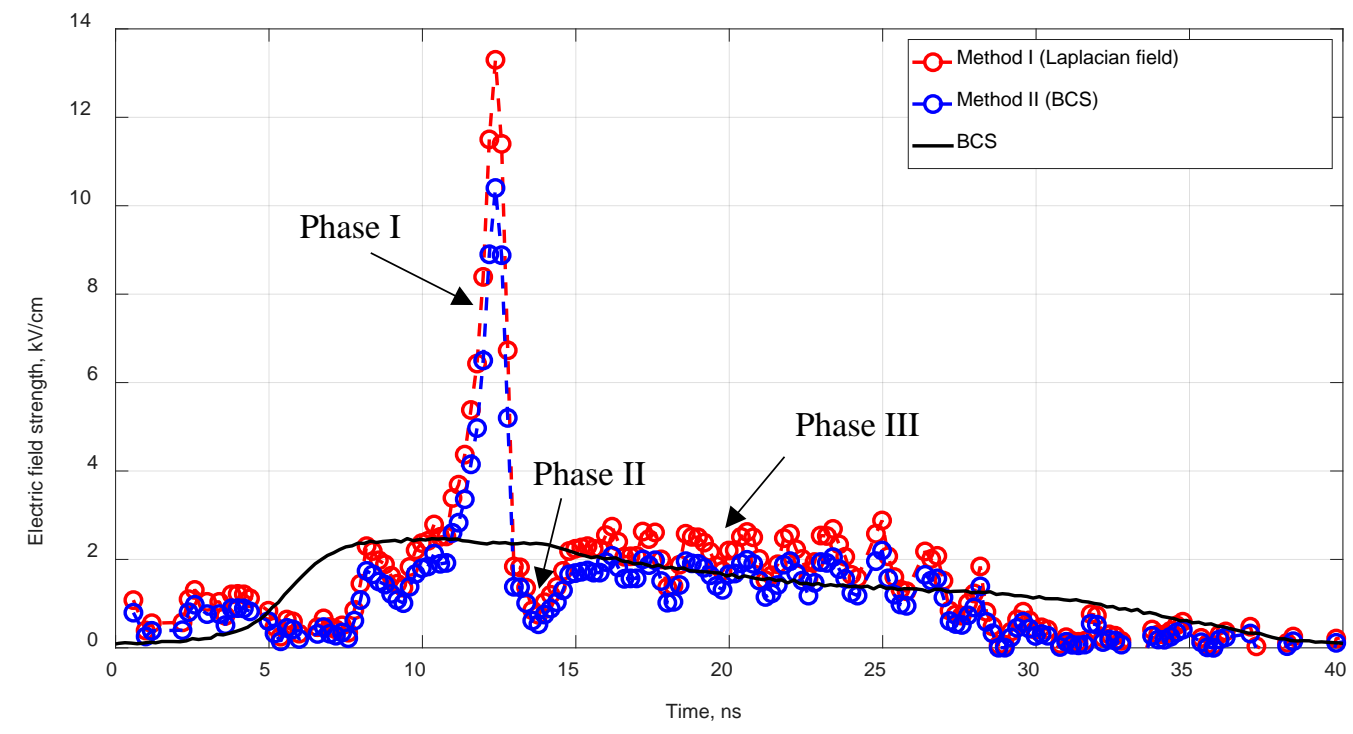

Fig. 5 Electric field evolution obtained in the fast ionization wave, nanosecond pulse discharge in pure $\mathbf{N}_{2}$ at a pressure of 20 mbar. Method I uses the E-FISH signals obtained in the Laplacian field for calibration, while method II makes use of the BCS field values when the discharge gap is 'closed'. The 3 distinct phases of the electric field evolution are labelled phase I, II and III respectively.

\section{B. $\underline{\text { N-atom TALIF Experiments }}$}

Based on [12], the number density of atomic nitrogen may be written as:

$$
n_{N}=\left(\frac{\sigma_{K r}}{\sigma_{N}}\right)\left(\frac{v_{N}}{v_{K r}}\right)^{2}\left(\frac{P M T_{K r}}{P M T_{N}}\right)\left(\frac{a_{K r}}{A_{K r}}\right)\left(\frac{A_{K r}}{A_{N}}\right)\left(\frac{F_{K r}}{F_{N}}\right)\left(\frac{A_{N}+Q_{N}}{A_{K r}+Q_{K r}}\right)\left(\frac{I_{K r}}{I_{N}}\right)^{2}\left(\frac{S_{N}}{S_{K r}}\right) n_{K r}
$$

where $n$ is the number density, $\sigma$ is the two-photon absorption cross-section, $v$ is the resonance frequency, $P M T$ is the photomultiplier quantum efficiency at the resonant wavelength(s), $a$ is the natural radiative rate for a specific fluorescence transition, $A$ is the combined natural radiative rate from a particular upper level, $F$ is the filter transmission, $Q$ is the quenching rate of the two-photon excited state, $I$ is the laser intensity and $S$ is the spectrally and temporally integrated fluorescence measured by the photomultiplier. Subscripts $\mathrm{N}$ and $\mathrm{Kr}$ refer to atomic nitrogen and krypton respectively. The ratio of two-photon cross-sections (value of 0.67) is obtained from [12]; PMT quantum efficiencies and filter transmission curves are obtained or interpolated from their respective manuals. The natural radiative rate of $\mathrm{N}$ (value of $29.6 \mathrm{~ns}$ ) is obtained from [12], while the optical branching ratio in the absence of quenching $\left(\frac{a_{K r}}{A_{K r}}\right)$ is assigned a value of 0.962 based on an average of the values found in [1214]. The reference $\mathrm{Kr}$ number density of $6.7 \times 10^{16} \mathrm{~cm}^{-3}$ is obtained by flowing $\mathrm{Kr}$ gas at room temperature through the capillary at a pressure of 2.7 mbar. In spite of the use of a nanosecond laser source, all the acquired TALIF signals have decay rates $(A+Q)$ which are well-resolved and substantially longer than the 6.5 ns duration of the UV laser pulse (see fig. 8 later). This allows quenching corrections to be directly implemented.

To ensure operation within the 'linear' regime, defined as when the fluorescence signal $S$ observes a quadratic dependence on the laser intensity (see eqn. (2)), the laser energy is varied over a range of 2-300 $\mu \mathrm{J}$ in order to establish the optimum laser intensity. The energies used for the $\mathrm{N}$ and $\mathrm{Kr}$ experiments are $200 \mu \mathrm{J}$ and $10 \mu \mathrm{J}$ respectively.

Figure 6 shows the absolute $\mathrm{N}$-atom density measured in the late afterglow. Due to the presence of strong fluorescence-overlapping plasma emission produced during each of the discharge pulses, good signals were only possible $5 \mu$ s after the initiation of the discharge. The N-atom density remains relatively constant up to $100 \mu \mathrm{s}$ after the discharge and displays a peak value of about $5.5 \times 10^{12} \mathrm{~cm}^{-3}$ or a corresponding ionization degree of about $10^{-5}$. This value of the $\mathrm{N}$-atom density is considered reasonable given the low specific deposited energy of this discharge. The total specific energy deposited by the 3 high-voltage pulses is measured to be about 0.01 $\mathrm{eV} /$ molecule as shown in fig. 7 . In addition, fig. 8 shows that the evolution of the TALIF decay rates fall within the range reported in the literature for quenching rate coefficients measured at $300 \mathrm{~K}$. This indicates that the temperature of the discharge is close to that of the ambient, in agreement with what might be expected at these low levels of specific deposited energy. 
It is worth mentioning that the current levels of $\mathrm{N}$-atom density are about 4-5 orders of magnitude lower than that measured in a capillary $(d=1.7 \mathrm{~mm})$ under virtually the same operating conditions [11]. The main difference is that the specific deposited energy in the capillary, due to its smaller volume, is much higher ( $\approx 2 \mathrm{eV} / \mathrm{molecule}$ ). This reaffirms the importance of the specific deposited energy in determining the efficiency of the atomic species production for this class of discharges.

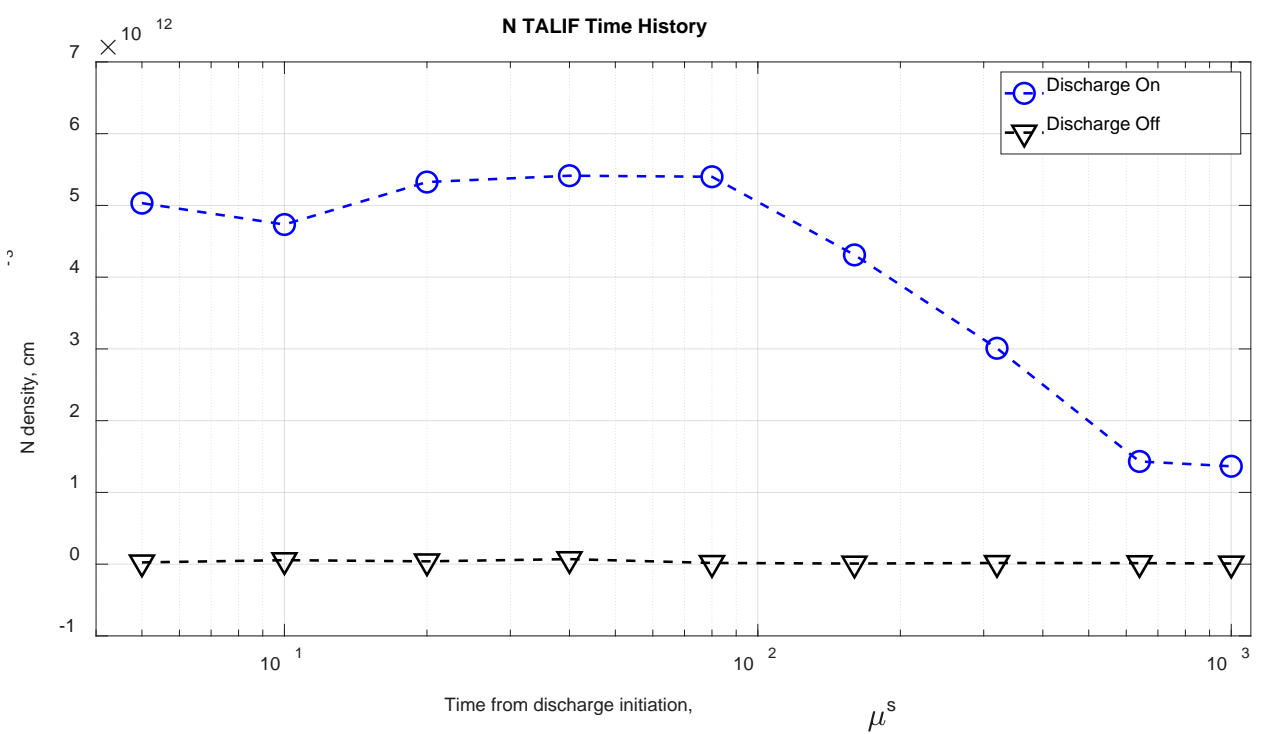

Fig. 6 Time evolution of $\mathrm{N}$-atom density measured using TALIF in pure $\mathrm{N}_{2}$ and a pressure of 20 mbar.
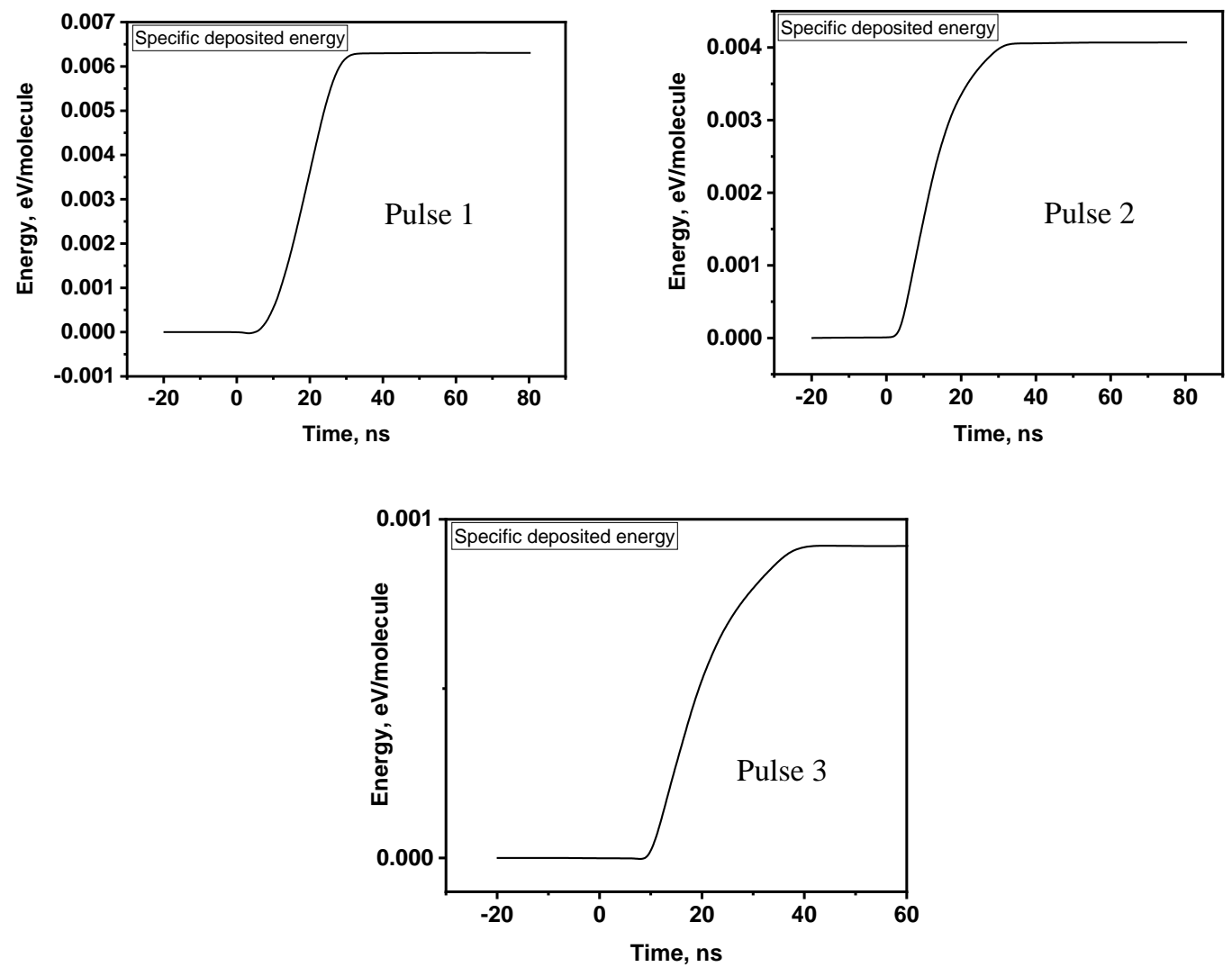

Fig. 7 Specific deposited energy for each high voltage pulse as measured from the BCSs. Pulse 1: 0.0063 eV/molecule, pulse 2: $0.0041 \mathrm{eV} / \mathrm{molecule,} \mathrm{pulse} \mathrm{3:} 0.0009 \mathrm{eV} / \mathrm{molecule.} \mathrm{Total} \mathrm{specific} \mathrm{deposited} \mathrm{energy:}$ $0.011 \mathrm{eV} / \mathrm{molecule}$ 


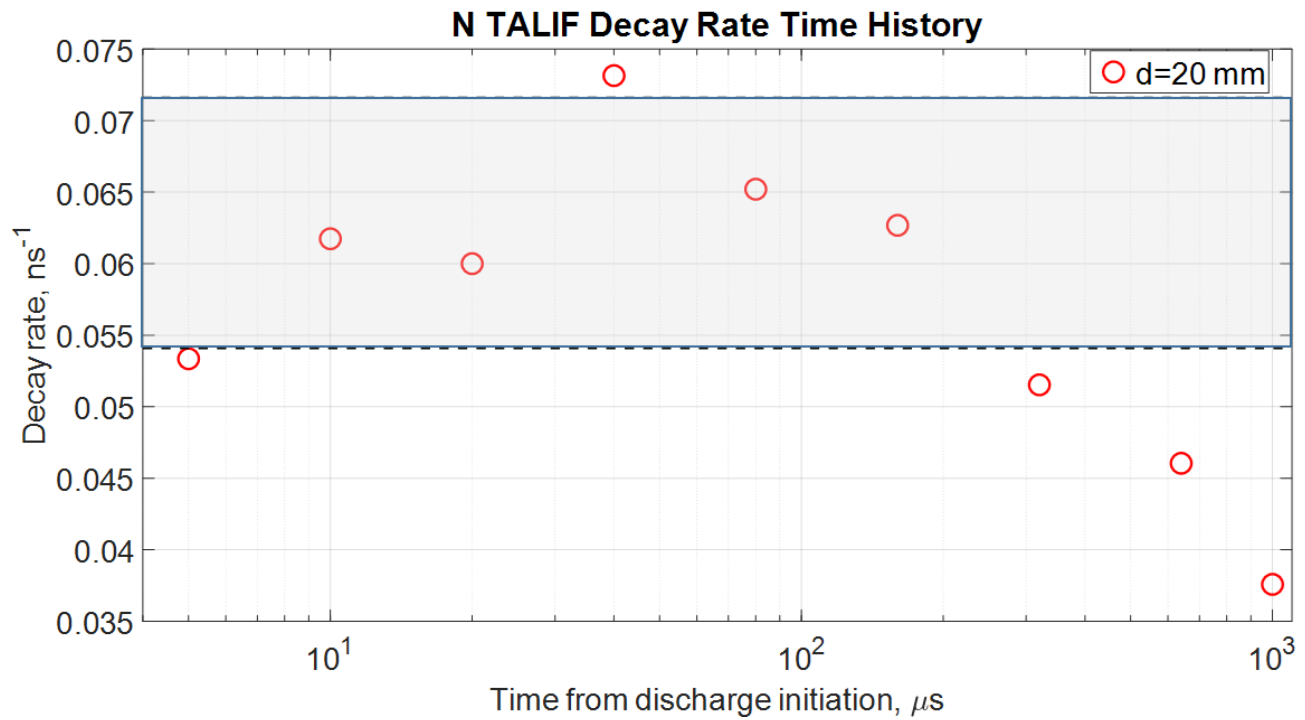

Fig. 8 Time evolution of $\mathrm{N}$-atom TALIF decay rates in pure $\mathrm{N}_{2}$ and a pressure of 20 mbar. The measured rates are relatively constant and fall within the range predicted using room temperature $(300 \mathrm{~K})$ quenching rate coefficients within the literature. This range is indicated by the grey shaded region.

Another source of complexity related to this discharge is the issue of radial non-uniformity, particularly with regards to modeling efforts. Figure 9a shows a representative two-dimensional emission image captured with an ICCD camera about $5 \mathrm{~ns}$ after the first high-voltage pulse is delivered. The spectral content of this emission, which is anticipated to originate from the second positive system of $\mathrm{N}_{2}$, displays a pronounced non-uniformity, increasing outwards from the center of the tube towards the walls. This is more clearly seen if one considers a radial emission profile obtained from a vertical slice at any chosen location along the length of the tube. Such a profile is shown in fig. 9b. Since these emission images are path integrated, an inverse Abel transform is performed in order to recover the true radial profile. The results of this transform shows that the emission at the center of the tube is only a few percent of that near the tube wall. Furthermore, the nature of the inverse Abel procedure also imposes a significant uncertainty on the precise value of the emission at the tube center relative to the periphery. This turns out be a severe problem for simulations, since (in addition to the transmitted current) a copy of the radial emission profile is used as an input for the initial electron density [8]. Since the initial electron density profile has a strong influence on the plasma chemistry, any uncertainty in the emission data translates into a possible error in the predictions for consequent $\mathrm{N}$-atom production.

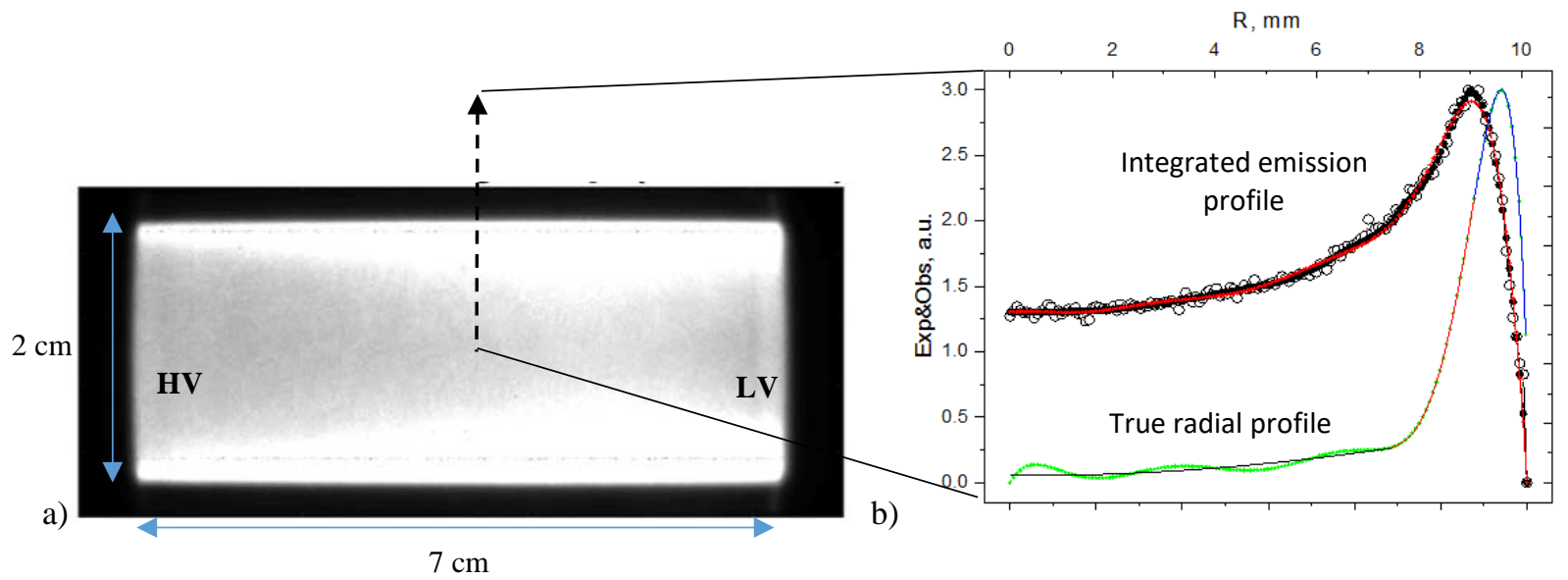




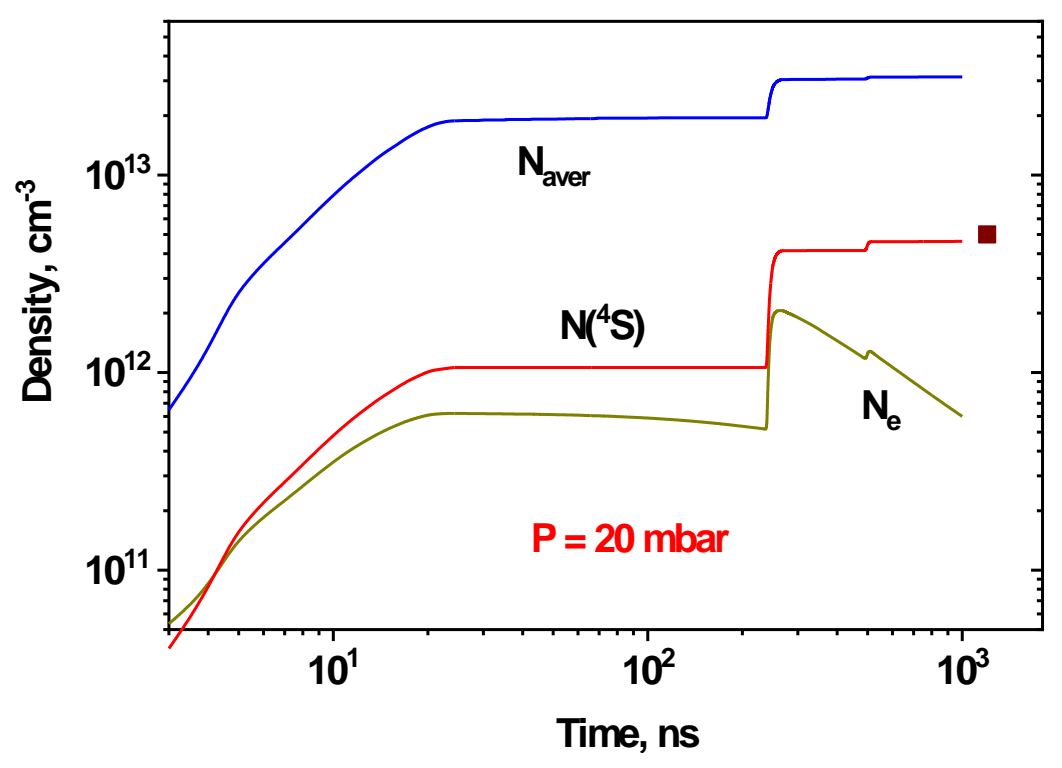

Fig. 9 a) Two-dimensional image of the $20 \mathrm{~mm}$ tube discharge illustrating the radial non-uniformity of the emission. b) Integrated and true radial emission profile corresponding to a vertical slice taken at mid length of the tube. c) Time evolution of electron and $\mathrm{N}$-atom density based on numerical predictions. The blue curve is obtained by averaging the $\mathrm{N}$-atom density over the entire tube. The red curve represents the $\mathrm{N}$ atom density at the tube center. (A representative experimental data point is indicated by a red square.)

Figure 9c shows the simulated temporal evolution of the N-atom production. In these simulations, the specific deposited energy is artificially weighted by the corresponding emission data along the radius of the tube, so as to account for the radial non-uniformity. In particular, it is noted that the agreement between experiments and simulations is much better if comparisons are made with predictions at the discharge axis versus an average over the entire tube. This serves to emphasize the sensitivity of the modelling predictions to the spatial uniformity of the discharge.

\section{Conclusions and Future Work}

The electric field strengths and $\mathrm{N}$-atom production have been measured in a fast ionization wave, nanosecond pulse discharge in pure $\mathrm{N}_{2}$ at 20 mbar using E-FISH and TALIF. The results of the E-FISH experiments capture the existence of 3 distinct phases during the development of the fast ionization wave with a time resolution of about 200 ps, and further underlines the usefulness of the E-FISH method as a tool for making sub-ns measurements in rapidly evolving fields. The TALIF measurements in the post discharge show a peak N-atom density of about $5.5 \times 10^{12} \mathrm{~cm}^{-3}$, within reasonable expectations of a discharge with a relatively low specific deposited energy on the order of $0.01 \mathrm{eV} /$ molecule. Future work will focus on verifying the origin of the E-FISH signal and also continue to examine the dependence of the $\mathrm{N}$-atom density on the reduced electric field by varying the geometric dimension (both length and diameter) of the discharge tube.

\section{Acknowledgments}

The work is partially supported by the following funding sources: Ecole Polytechnique-DGA 2790, French National Research Agency, ANR (ASPEN Project), LabEx Plas@Par and the French-Russian international laboratory LIA KaPPA 'Kinetics and Physics of Pulsed Plasmas and their Afterglow' (including RFBR project 17-52-16001 and CNRS financial and organizational support). The experiments were conducted at the Centre Laser de l'Université Paris Sud (CLUPS/LUMAT FR 2764). The authors are thankful to Dr. Cyril Drag for the use of equipment essential for conducting this study, Ali Mahjoub, Bruno Dufour and Pascal Pariset for technical assistance, and to Dr Michel Broquier and Dr Gilles Grégoire for help in setting up these experiments. The support of Prof. Adamovich by the Ecole Polytechnique Gaspard Monge Visiting Professor (GMVP) Program is gratefully acknowledged. 


\section{References}

[1] Dogariu A, Goldberg B M, O’Byrne S and Miles R B 2017 Species-independent femtosecond localized electric field measurement Phys. Rev. Appl. 7024024

[2] Babich L P, Lo 1ko T V and Tsukerman V A 1990 High-voltage nanosecond discharge in a dense gas at a high overvoltage with runaway electrons Sov. Phys.-Usp. 33 521-40

[3] Anikin N B, Starikovskaia S M and Starikovskii A Y 2002 Polarity effect of applied pulse voltage on the development of uniform nanosecond gas breakdown J. Phys. D: Appl. Phys. 352785

[4] Anikin N B, Starikovskaia S M and Starikovskii A Y 2004 Study of the oxidation of alkanes in their mixtures with oxygen and air under the action of a pulsed volume nanosecond discharge Plasma Phys. Rep. 301028

[5] Klochko A V, Starikovskaia S M, Xiong Z and Kushner M J 2014 Investigation of capillary nanosecond discharges in air at moderate pressure: comparison of experiments and 2D numerical modelling J. Phys. D: Appl. Phys. 47365202

[6] Darny T, Pouvesle J-M, Puech V, Douat C, Dozias S and Robert E 2017 Analysis of conductive target influence in plasma jet experiments through helium metastable and electric field measurements Plasma Sources Sci. Technol. 26 045008

[7] Duvillaret L, Rialland S and Coutaz J 2002 Electro-optic sensors for electric field measurements. I. Theoretical comparison among different modulation technique J. Opt. Soc. Am. B 19 2692-703

[8] Lepikhin N D, Popov N A, and Starikovskaia S M 2018 Fast gas heating and radial distribution of active species in nanosecond capillary discharge in pure nitrogen and N2: O2 mixtures. Plasma Sources Science and Technology, 27(5), 055005.

[9] Goldberg B M, Chng T L, Dogariu A and Miles R B 2018 Electric field measurements in a near atmospheric pressure nanosecond pulse discharge with picosecond electric field induced second harmonic generation Appl. Phys. Lett. 112 064102

[10] Simeni Simeni M, Tang Y, Frederickson K and Adamovich I V 2018 Electric field distribution in a surface plasma flow actuator powered by Ns discharge pulse trains Plasma Sources Sci. Technol. 27104001

[11] Chng T L, Lepikhin N D, Orel I, Popov N A and Starikovskaia S M 2019 Atomic Nitrogen Density Measurements in a Nanosecond Capillary Discharge. In AIAA Scitech 2019 Forum (p. 0745).

[12] Niemi K, Schulz-Von Der Gathen V, Döbele HF. Absolute calibration of atomic density measurements by laser-induced fluorescence spectroscopy with two-photon excitation. Journal of Physics D: Applied Physics. 2001 Jul 17;34(15):2330.

[13] Chang R S, Horiguchi H, Setser D W. Radiative lifetimes and two-body collisional deactivation rate constants in argon for $\operatorname{Kr}\left(4 p^{5} 5 p\right)$ and $\operatorname{Kr}\left(4 p^{5} 5 p^{\prime}\right)$ states. The Journal of Chemical Physics. 1980 Jul 15;73(2):778-90.

[14] Kramida, A., Ralchenko, Yu., Reader, J., and NIST ASD Team (2019). NIST Atomic Spectra Database (ver. 5.7.1).

[15] Wagenaars E, Bowden MD, Kroesen GM. Measurements of electric-field strengths in ionization fronts during breakdown. Physical review letters. 2007 Feb 14;98(7):075002.

[16] Ito T, Kobayashi K, Czarnetzki U, Hamaguchi S. Rapid formation of electric field profiles in repetitively pulsed highvoltage high-pressure nanosecond discharges. Journal of Physics D: Applied Physics. 2010 Jan 26;43(6):062001.

[17] Lomaev MI, Beloplotov DV, Tarasenko VF, Sorokin DA. Breakdown features of a high-voltage nanosecond discharge initiated with runaway electrons at subnanosecond voltage pulse rise time. IEEE Transactions on Dielectrics and Electrical Insulation. 2015 Aug 11;22(4):1833-40. 